\title{
Fingerprint Identification System: Non-zero Effort Attacks for Immigration Control
}

\author{
Fatai Olawale W. \\ Department of Computer Science \\ University of llorin, \\ Ilorin, Kwara State
}

\author{
Oluwade Bamidele A. \\ Department of Computer Science \\ University of llorin, \\ Ilorin, Kwara State
}

\author{
Awotunde Joseph B. \\ Department of Computer Science \\ University of llorin, \\ Ilorin, Kwara State
}

\begin{abstract}
A growing security issue today in Nigeria is the increased occurrence of identity fraud. Research shows that perpetrators of this act are foreigners who enter the country without any document and are employed as security officers, thereby posing security treats to lives and properties. It has been noted that these foreigners device a means of beating security devices put in place at the border. The Nigerian Immigration uses Automated Fingerprint identification system (AFIS) which is minutiae base and it's less noise tolerant unlike the correlation base approach. This paper therefore proposes a novel fingerprinting method that can help identify identity fraud. The method combines two approaches namely minutiae and correlation approaches, the idea is to see how the shortcomings of one is complemented by the other and the two approaches processes extraction and matching so as to get good and reliable images. Each of the approaches computes the matching score and the mean of the two resulting scores is taken. The mean is compared with the established threshold such that the system provides response by indicating whether the verification is successful or not. It follows that the adoption of this new method by organizations like the Nigerian Immigration Service will drastically reduce, if not totally eradicate, the level of insecurity in the country.
\end{abstract}

Keywords and Phrases:

Security, Immigration, Control, Identity fraud, Threshold

\subsection{INTRODUCTION}

Fingerprint as been one of the core methodologies for person identification, It remains a reliable, efficient and commonly accepted biometric. Fingerprint databases are in use worldwide for the purposes of personal identification, border control as well as to facilitate criminal forensic investigation. Many countries have multiple fingerprint databases, with each database serving a specific purpose and sometimes they complement each others.

It is established that terrorist activities have negative effect on National development in Nigeria [8]. The Immigrant seen as the real threats are those who cross the borders without valid documents. Recent development in the security situation in Nigeria shows that terrorist groups are predominantly foreigners from neighbouring countries. This development posse's security threats due to poor border control. Nigerian Immigration Service uses AFIS which is minutiae based and it's less noise tolerant thereby producing a blur images in some cases.

Minutiae-based methods are the most popular ones being included in almost all contemporary fingerprint identification and verification systems [7]. This method represents the fingerprint by its local features like, terminations and bifurcations called as minutia. Minutiae are small points of interest in the fingerprint image. The minutiae-based method does the recognition in two stages that is minutiae extraction and minutiae matching [9]. But the cross correlation based technique is a promising approach to fingerprint authentication for the new generation of high resolution and touch less fingerprint sensors.

The provided a fingerprinting method which combines two approaches namely minutia and correlation approaches for the process of extraction and authentication, the idea is to see how the shortcomings of one can make up for the other. It also follows that efforts should be made to reduce terrorist activities by Government through appropriate sanction and machines (fingerprint device) put in place at the borders i.e. tackling of inherent challenges through integration of biometric-based technology into Nigeria immigration system.

In this paper the authors will examine the features of both the minutia and correlation approaches for the process of extraction and authentication and see how both methods can complement each others.

\subsection{RELATED WORK}

Fingerprinting has been the most widely used during the 20th century. The maturity of Biometric techniques and mainly the dramatic improvement of the captured devices have led to the proposal of fingerprinting in multiple applications but in the last years, minutiae have been the main type of algorithm used. The minutiae are relatively stable and robust to contrast, image resolution and global distortion as compared to other fingerprint representation. However, to extract minutiae from a poor quality image is not an easy task [1]. The aim of combining minutiae and correlation techniques is to improved robustness, completeness, usability, and efficiency.

Nigeria could be said to be much more vulnerable to the threats than any country in Africa. Being the largest population and market in Africa (over 140 million people), peoples of different backgrounds enter the country for socio-economic purposes from all over the world [2]. Almost all the migration and security stakeholders seemed to agree that its borders are leaky and poorly monitored. Also almost on the daily basis would one read or hear of migration-related offences committed by Nigerians and non-Nigerians within and outside its borders respectively [2].

A fingerprint is a pattern of ridges and furrows located on the tip of each finger. Fingerprints were used for personal identification for many centuries and the matching accuracy was very high [3]. Patterns have been extracted by creating an inked impression of the fingertip on paper. Today, compact sensors provide digital images of these patterns. Fingerprint recognition 
for identification acquires the initial image through live scan of the finger by direct contact with a reader device that can also check for validating attributes such as temperature and pulse. In real-time verification systems, images acquired by sensors are used by the feature extraction module to compute the feature values. The feature values typically correspond to the position and orientation of certain critical points known as minutiae points [3].

Fingerprinting has been the most widely used during the $20^{\text {th }}$ century. The maturity of Biometric techniques and mainly the dramatic improvement of the captured devices have led to the proposal of fingerprinting in multiple applications but in the last years, minutiae have been the main type of algorithm used. The minutiae are relatively stable and robust to contrast, image resolution and global distortion as compared to other fingerprint representation. However, to extract minutiae from a poor quality image is not an easy task [1].

[1]. Proposed a local correlation based fingerprint matching algorithm. In the proposed algorithm the author used a window size of $42 \times 42$ pixels around the minutia locations in the template image and $32 \times 32$ pixels size windows around the corresponding location in the query image. The normalized cross-correlation between the query window and template window is computed and peak is detected. If the peak lie outside 10 pixels from the centre, the correlation between template and query window is zero otherwise this is the absolute value of correlation between the query and template window. The local correlation of all template windows with the corresponding regions in the query image are computed and mean correlation value is found. In this way all the possible correspondence from the alignment stage are tested and maximum correlation value over all the correspondence is taken as the matching score between the query and template image.

The aims of combining minutiae and correlation techniques are to improved robustness, completeness, usability, and efficiency.

\subsection{RESEARCH METHODOLOGY}

Fingerprint verification and identification systems follow minutiae-based approach. The Nigerian Immigration Service make use of AFIS which is minutia based, minutiae based algorithm has fixed radius and so can tolerate missing and spurious minutiae better than nearest neighbour based approach. Also minutiae are error tolerant too therefore makes coding a smooth one. However, minutia based technique are not noise tolerant as blurry images can be produced when noise interferes with it. This paper aims at trying to see how minutiae algorithm and correlation algorithm can be combined in a setting like the Nigerian Immigration for extraction and matching processes. For the purpose of this paper the mean of the score from the two approaches will be taken as the final score that would be compared with the established so as to increase FAR of an impostor before an acceptance or rejection is established.

\subsection{OVERVIEW OF THE FRAMEWORK}

This section of the paper describes the overview framework of the methodology used for the proposed system.

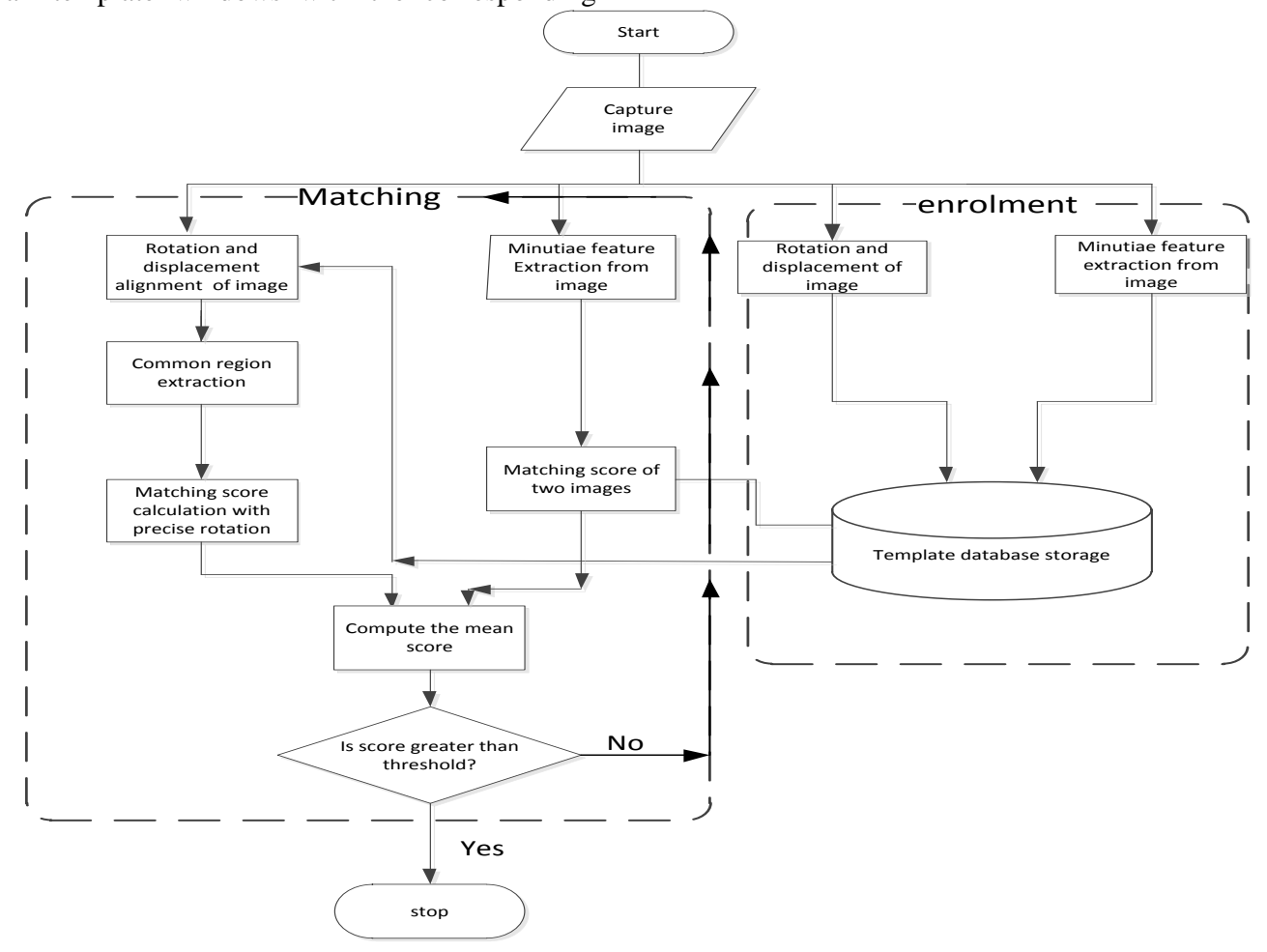

Figure 3.1 Flowchart

The figure above shows the overview of the Framework for the Biometric Recognition system (Flowchart) which combines two approaches namely minutia and correlation algorithm. From the above flowchart it will be noted that the flowchart consists of two main parts namely:

- $\quad$ Enrolment
- Matching

\subsubsection{Enrolment:}

The enrolment parts consist of three items which are minutiae feature extraction from image, rotation and displacement of images and template database storage. 


\subsubsection{Minutiae feature extraction from}

image: This forms the part where minutiae extraction is done and of course processes like binarization, thinning and bifurcation would be done in this part.

\section{Binarization}

The process of binarization is converting grayscale image to binary form is known as binarization. In a gray-scale fingerprint image, a pixel can take on 256 different intensity levels [4]. The threshold value is used to convert greyscale image to binary. The pixel values below the threshold are set to zero and the intensity values greater than the threshold is assigned one. In binary image the pixel values are assigned 0 and 1 to black and white pixels respectively. The processing of binary image is easy as it has only two intensity levels compared to gray scale image of 256 intensity levels [4]. Figure 3.1 shows original fingerprint image and its corresponding binarized image. The disadvantage of binarization is that the ridges end near the boundary is considered as minutia even though it is not actual minutia. The problem of binarization is eliminated in thinning process.

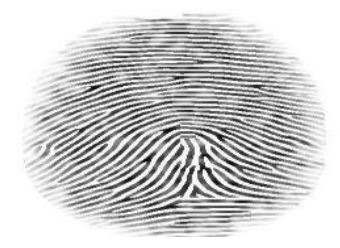

(a)

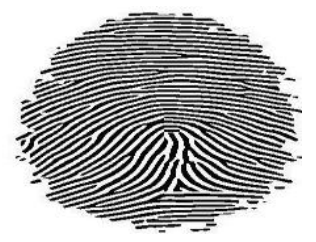

(b)
Figure 3.1 (a) Original Fingerprint (b) Binarized image Image source: [5].

\subsubsection{Thinning}

Thinning is the process of reducing the width of each of the ridges pixel of the same size and it is done on binarized image to reduce the thickness of all ridge lines to a single pixel width so as to extract minutiae points effectively. This is done with the implementation of block filter, block Filter is used to scans the image at the boundary to preserves the quality of the image and extract the minutiae from the thinned image, the location and orientation of minutiae points does not change during thinning when compared to original fingerprint that is, it ensures accurate estimation of minutiae points. The outermost pixels is preserved during thinning by placing white pixels at the boundary of the image, as a result first five and last five rows, first five and last five columns are assigned value of one. Dilation and erosion thickens the area of the valleys in the fingerprint as a result the ridges are effectively eroded. A binarized Fingerprint and the image after thinning are shown in Figure 3.2.

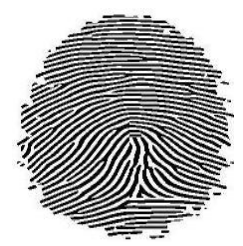

(a)

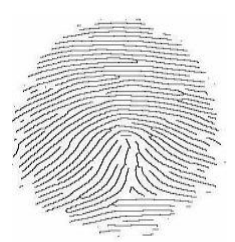

(b)
Figure: Binarized fingerprint Image after thinning Image source: [5].

\section{Minutiae Extraction}

The minutiae location and the minutiae angles are derived after minutiae extraction. The terminations which lie at the outer boundaries are not considered as minutiae points, and Crossing Number is used to locate the minutiae points in fingerprint image. Crossing Number is defined as half of the sum of differences between intensity values of two adjacent pixels. The dark rectangles are regarded as 1 and the blank rectangles are regarded as zero since it has undergone binarization which is conversion to zeros and ones. If crossing Number is 1, 2 and 3 or greater than 3 then minutiae points are classified as Termination, Normal ridge and Bifurcation respectively, is shown in figure 3.3

\begin{tabular}{|l|l|}
\hline & \\
& \\
Crossing Number =2. \\
Normal ridge pixel.
\end{tabular}

Figure3.3 crossing number and type of minutia Image source: [5]. 
To calculate the bifurcation angle, we use the advantage of the fact that termination and bifurcation are dual in nature. The termination in an image corresponds to the bifurcation in its negative image hence by applying the same set of rules to the negative image, we get the bifurcation angles. Figure 3.4 shows the original image and the extracted minutiae points. Square shape shows the position of termination and diamond shape shows the position of bifurcation as in figure 3.4 (b)

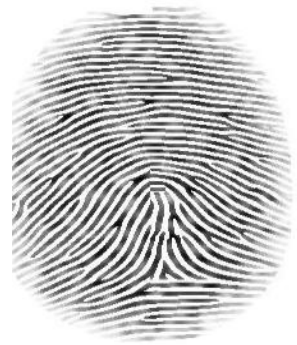

(a)

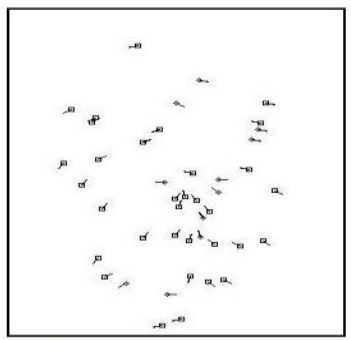

(b)
Figure3.4 Gray-scale fingerprint Minutiae point Image source: [5].

\subsubsection{Minutiae Matching}

To compare the input fingerprint data with the template data Minutiae matching is used. For efficient matching process, the extracted data is stored in the matrix format. The data matrix is as follows. Number of rows: Number of minutiae points. Number of columns: 4

Column 1: Row index of each minutia point. ( $x$ Coordinate)

Column 2: Column index of each minutia point. ( $y$ Coordinate)

Column 3: Orientation angle of each minutia point. (Minutiae angle $\theta$ of the particular minutiae point to be paired i.e input image and template image).

Column 4: Type of minutia. (A value of ' 1 ' is assigned for termination, and ' 3 ' is assigned for bifurcation). In other words $(x, y, \theta$ and the minutiae type of input template would be paired with the same of $x, y, \theta$ and the minutiae type of registered template).

During the matching process, each input minutiae point is compared with template minutiae point considering the above properties i.e ( $x, y, \theta$ and the type of minutiae). In each case, template and input minutiae are selected as reference points for their respective data sets. The reference points are used to convert the remaining data points to polar coordinates. Equation (4) is used to convert the template minutiae from row and column indices to polar coordinates.

$$
\left[\begin{array}{c}
r_{k}^{T} \\
\emptyset_{k}^{T} \\
\theta_{k}^{T}
\end{array}\right]=\left[\begin{array}{c}
\sqrt{\left(\operatorname{row}_{k}^{T}-\operatorname{row}_{\text {ref }}^{T}\right)^{2}+\left(\operatorname{col}_{k}^{T}-\operatorname{col}_{\text {ref }}^{T}\right)^{2}} \\
\tan ^{-1}\left(\frac{\text { row }_{k}^{T}-\operatorname{row}_{r e f}^{T}}{\operatorname{col}_{k}^{T}-\operatorname{col}_{\text {ref }}^{T}}\right) \\
\theta_{k}^{T}-\theta_{\text {ref }}^{T}
\end{array}\right] \text {--- (4) }
$$

$$
\begin{array}{ll}
r_{k}^{T}= & \text { Radial distance of } \mathrm{k}^{\text {th }} \text { minutiae. } \\
\emptyset_{k}^{T}= & \text { Radial angle of } \mathrm{K}^{\text {th }} \text { minutiae } \\
\theta_{k}^{T}= & \text { Orientation angle of } \mathrm{K}^{\text {th }} \text { minutiae }
\end{array}
$$

$\operatorname{Row}_{r e f}^{T}, \operatorname{col}_{r e f}{ }^{T}=$ row index and column index of reference points currently being considered.

Similarly the input matrix data points are converted to polar coordinates using the Equation (5)

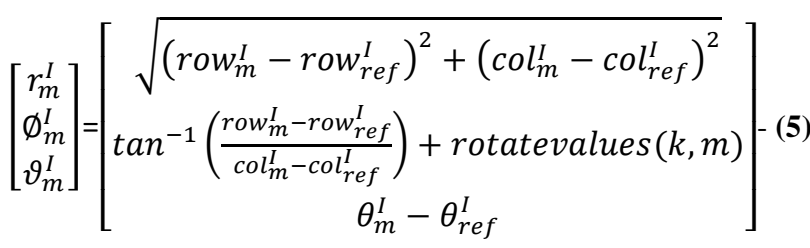

Rotate values $(k, m)$ represents the difference between the orientation angles of $T k$ and $I m$. Tk and $I m$ represent the extracted data in all the columns of row $\mathrm{k}$ and row $\mathrm{m}$ in the template and input matrices, respectively.

\subsubsection{Minutiae Algorithm}

Input: Gray-scale fingerprint image.

Output: Verified fingerprint image with matching score.

$>$ Fingerprint is binarized

$>$ Thinning on binarized image

$>$ Minutiae points are extracted. Data matrix is generated to get the position, orientation and type of minutiae.

> Matching of live test fingerprint with template

$>$ Matching score of two images is computed, if matching score is greater than the threshold then it stops else it returns to enrollment

\subsubsection{Rotation and displacement of image:}

This forms the part where the image is normalized to get an authentic and reliable image to be stored in the database so as to aid the process of matching.

3.1.1.3Template database storage: This part stores data that is, all the templates and information that are been generated from the process of minutiae extraction and rotation and displacement of image. 


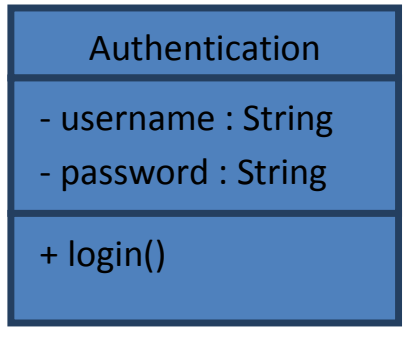

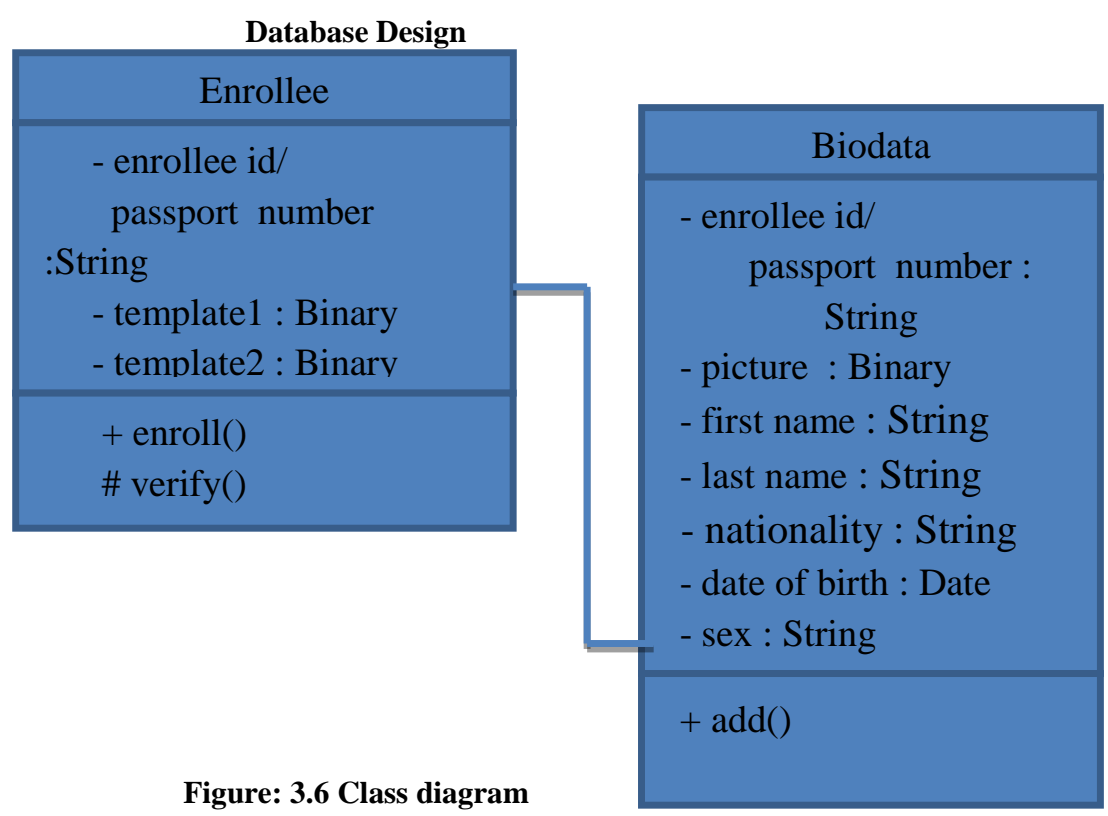

The above diagram represents the database design and structure which contains three major parts which are enrollee, biodata and authentication

Enrollee: this describes the enrollee that is contains the enrollee's ID which is sub-divided into passport number which can take string characters, template 1 and 2 which can take binaries, images during capturing, extraction and verification which takes binaries

Biodata: This is the part that describes the biodata been stored in the database and it comprises of passport number which can take string characters, picture of the enrollee which can take binary characters, first name which can take alphabetic characters, last name which can take alphabetic characters as well Nationality of the enrollee which can also take alphabetic and string characters, date of birth which can take string characters date issued and expiry date can take string characters too.

Authentication: this is the part of the database that allows access into and out of the database, this part monitors the kind of people that uses the database and controls unwanted users and unnecessary logins and access into the database for more secured and protected database environment.

3.2.1 Matching: This part also consist of two main parts the minutiae matching part and the rotation and displacement of image matching part (correlation)

3.2.2. Minutiae matching: Once the minutia feature extraction is done the next phase is to compare the live template with the stored template, the system fetches the template from the template database storage and compares it with the live template. Once the matching is done, the matching score from the two templates is computed.

\subsubsection{Rotation and displacement of aligned image:}

There is need to normalize the rotation and displacement between registered fingerprint image and input fingerprint image in order to perform high accuracy fingerprint matching in this stage the researcher has the normalized image.

\subsubsection{Common Region:}

In this stage the researcher extracts the overlapped region that is, the intersection of the two images and only the effective image areas of $f(n 1, n 2)$ and $g(n 1, n 2)$, with the same size are extracted for use in succeeding image matching steps. This stage helps to improve the accuracy of matching.

\subsubsection{Matching score calculation with precise rotation:}

In this stage the researcher generates the sets of rotated replicas of $f \theta(n 1, n 2)$ of $f(n 1, n 2)$ over the angular range of $-2^{\circ} \leq$ $\theta \leq 2^{\circ}$ with an angle of 0.5 and calculate BLPOC function rK1K2 $f \theta(n 1, n 2)$ then the correlation peak can be observed at the centre of the BLPOC function thus the matching score is defined between the two images as the sum of the highest $P$ peaks of the BPLOC function

\subsubsection{Compute the mean score:}

At this stage the score gotten from the matching of the two techniques are been added up and their mean is gotten by dividing the result gotten from the addition by 2 . The resulting score is taken as the matching score.

Hence the resulting score is compared with the established set threshold score, if the score is greater than the threshold it concludes template match and it stops otherwise it indicates mismatch and it returns to matching and takes the process again.

\subsection{DISCUSSION AND RESULT}

For the purpose of this two algorithms are combined, the soul idea behind this concept is to see how the shortcomings of one algorithm is been made up for by the other algorithm therefore the process of verification is done by entering the enrollee's passport identification number and the enrollee is asked to take the verification process by placing his/her finger on the biometric fingerprint scanner (Secugen), it is captured and the 
admin verifies the live biometric fingerprint captured with the one in the database. Each approach (correlation techniques image matching and minutiae-based matching) computes its matching score and the mean of the score from the two approaches is compared with the established set threshold score and if the resulting score from the matching is greater than the threshold score the system displays "VERIFICATION SUCCESSFUL" and the enrollee's passport is displayed together with the address and phone number to confirm that the enrollee is actually the person he/she claims to be but if the resulting score is less than the established threshold the system ignores and displays "WRONG USER"

\subsection{CONCLUSION AND FUTURE WORK}

This paper has been able to achieve some far-reaching objectives of dealing with illegal immigrants entering the country without valid documents and using some tactics and fraudulent acts to beat the machines put in place (fingerprint scanner) to check them while entering the country. By providing a reliable extraction and matching processes by combining two techniques namely, minutiae and correlation for the processes. The sole idea behind this is that one technique makes up for the deficiency of the other by so doing we obtain a reliable and efficient result and provide a more secured environment where immigrants will not be able to beat the system with their fraudulent tactics or act as it use to be before now. If this system is applied in the Nigerian Immigration settings, illegal immigrants will not be nabbed if they try to play pranks or find their way around beating the machine put in place to check immigrants coming into the country.

For the purpose of this paper the authors intends to build more on the streamlined and hybridized algorithm and the use of fingerprint to use in checking in immigrants into the country, in other words the authors were able to combined minutiae and correlation algorithms for a better methods for verification because the deficiency in one makes up for the other. Further work can be done on bimodal biometric by combine two biometric i.e. fingerprint and face recognition in verification and identification of immigrants.

\section{Reference:}

[1] B. Roli, S. Priti \& B. Punam, (2011). Minutia Extraction from Fingerprint Image: A review, International Journal of Computer Science Issues Vol. 8, Issue 5, No3, “001.

[2] Frank Laczko, Abye Makonnen (2009). Migration in Nigeria: Thematic Document 2009 National Security and Migration: towards an Effective Cross-border management in Nigeria, (C) 2009 International Organization for Migration (IOM), International Organization for Migration, 17 route des Morillons 1211 Geneva 19, Switzerland.

[3] ICAO, (2006). "Machine readable travel documents", Technical report, ICAO 2006.

[4] Jagadeesan, (2010). Secured Cryptographic key Generation from Multimodal Biometrics: Feature level Fusion of Fingerprint and Iris, (IJCSIS) International Journal of Computer Science and Information Security, Vol. 7, No. 1.

[5] J, K. Ravi, B. Raja, \& K. R Venugopal, (2009). International Journal of Engineering Science and Technology, Fingerprint recognition using minutia score Matching, Vol.1 (2), 35-42

[6] K. Nandakumar and A. K. Jain, (2004). Local Correlationbased Fingerprint Matching, Proceedings of Indian Conference on Computer Vision, Graphics \& Image Processing, pp.1-6, Kolkata.

[7] L. Hong, Y. Wan \& A. Jain, (1998). Fingerprint Image Enhancement: Algorithm and Performance Evaluation, IEEE Trans. Pattern Analysis and Machine Intelligence, vol. 20, no. 8, pp.777-789.

[8] Patric, Ebele and Chinedu, (2012). Terrorist activities and economic development in Nigeria: An early warning signal OIDA international Journal of sustainable development Vol 5, No.4, pp. 69-78, 2012.

[9] S. Prabhakar, A.K. Jain, J. Wang, S. Pankanti, \& R. Bolle, (2000). Minutia Verification and Classification for Fingerprint Matching, In Proc. ICPR2000, 15th Int. Conf. Pattern Recognition, Barcelona, Spain. 